\title{
Repeated Depression Screening During the First Postpartum Year
}

\author{
Barbara P. Yawn, MD, MSc \\ Susan Bertram, RN, MSN \\ Marge Kurland, RN, BSN \\ Peter C. Wollan, PbD \\ Olmsted Medical Center, Department of \\ Research, Rochester, Minnesota
}

\begin{abstract}
PURPOSE Postpartum depression (PPD) screening at 4 to 12 weeks' postpartum can improve outcomes for women when linked to in-practice management programs. The benefit of repeated PPD screening during the first year postpartum remains unclear.
\end{abstract}

METHODS We report a substudy of a large pragmatic trial of early PPD screening and practice management, the Translating Research into Practice for Postpartum Depression (TRIPPD) study. Outcome analyses were based on demographic information and Patient Health Questionnaire (PHQ-9) screening scores from questionnaires mailed to all enrolled women at baseline (4 to 12 weeks' postpartum) and again at 6 and at 12 months' postpartum. The main outcomes of this substudy were the 6-and 12-month rates of PHQ-9 scores that were 10 or greater for women whose baseline PHQ-9 scores were less than 10. Women whose scores were 10 or greater would be considered at high risk of PPD and appropriate for further evaluation.

RESULTS At 6 months, 134 (10.9\%) of the 1,235 women who did not have PHQ-9 scores greater than 10 at baseline had elevated scores appropriate for further evaluation. At 12 months, 59 (6.1\%) of the 969 women who did not have PHQ-9 scores greater than 10 at baseline or at 6 months had elevated scores. Together the 6- and 12-month repeated screenings identified 193 women at high risk of depression. This finding represents $13.5 \%$ of the 1,432 women whose screening results were negative for PPD at baseline.

CONCLUSIONS Repeated PPD screening at 6 and 12 months' postpartum increases the percentage of women identified as being at high risk of PPD. Further work will be required to understand the impact of this repeated screening on patient outcomes.

Ann Fam Med 2015;13:228-234. doi: 10.1370/afm.1777.

\section{INTRODUCTION}

M aternal postpartum depression (PPD) is common, costly, and associated with adverse outcomes for mothers, infants, and families. ${ }^{1,2}$ There is limited outcomes-based evidence to support universal or routine PPD screening in the early postpartum period. ${ }^{2-6}$ Neither the United States Preventive Services Task Force nor the American College of Obstetrics and Gynecology has made recommendations regarding routine PPD screening. ${ }^{7,8}$ The American Academy of Pediatrics, however, recommends universal screening in the early postpartum period and screening for parental depression at each well-child visit., ${ }^{9} 10$ The basis for those recommendations appears to be evidence that PPD is currently underrecognized, ${ }^{10,11}$ that screening increases recognition, ${ }^{10,12}$ and that pediatricians can discuss and refer women at risk for depression. ${ }^{10,13}$

The need to evaluate rescreening is based in part on the natural history of PPD as reported in a meta-analysis by Gaynes et al of 28 studies that included about 15,000 women. ${ }^{1}$ The prevalence of PPD diagnosis based on assessment by clinical interviews was $3.8 \%$ at 4 weeks, increasing to $4.7 \%$ at 12 weeks, and $5.6 \%$ at 26 weeks. Rates then fell to $1.0 \%$ at 32 weeks and 
$3.9 \%$ at 52 weeks. The increasing prevalence of PPD up to 6 months' postpartum suggests later-onset PPD. ${ }^{2}$

No study has evaluated the impact of outcomes related to repeated PPD screening, and only a few have assessed depression screening tool scores beyond the early postpartum period. ${ }^{14-20}$ Among those few studies, most report on special populations with small sample sizes and limited generalizability. ${ }^{14-19}$

We report data from a large PPD screening and management study ${ }^{4}$ that included baseline and repeated administration of the 9-item Patient Health Questionnaire (PHQ-9) ${ }^{21}$ on all women to determine rates of newly elevated PPD screening scores at 6 and 12 months' postpartum. We hypothesized that the rates would be greater than $10 \%$, supporting the need to add rescreening to future studies of the outcome of PPD screening programs.

\section{METHODS}

The data for this substudy came from a randomized pragmatic trial of PPD screening, follow-up, and management within family medicine practices, the Translating Research into Practice for Postpartum Depression (TRIPPD) study. The overall study design and main outcomes data have been published ${ }_{1}^{4}$ showing that primary care-based screening, diagnosis, and management improved maternal depression outcomes at 12 months.

The parent TRIPPD study enrolled 2,354 women across 16 states from 21 family medicine practices that provided maternity or early well-child care. The practices were randomized to routine PPD screening using the Edinburgh Postnatal Depression Scale (EPDS) at the 4 - to 12 -week postpartum or well-baby visit (more than $95 \%$ of eligible women were screened within the practice) or to continue providing usual care with no standardized PPD screening. More than $92 \%$ of eligible women, those who were 4 to 12 weeks' postpartum, aged 18 years or older, and could speak and write English or Spanish, were enrolled. Diagnosis and treatment occurred at the discretion of the woman's physician, guided by tools given to the intervention site practices and existing knowledge in the usual care sites. The study was approved by the institutional review boards for all sites, as well as by those of the investigators' home institutions. Outcomes data were collected by reports from the women and medical record reviews.

Study data came primarily from packets of outcome questionnaires given to the women at the time of enrollment and sent to their homes at 6 and 12 months' postpartum. Completed questionnaires were returned to the study's coordinating center (the Olmsted Medical Center, Department of Research). Each packet at the 3 time points included the PHQ-921 and the EPDS instruments, as well as questions regarding data on race, income, education, marital status, parity, number of adults living in the home, and being uninsured at any time postpartum. Insurance status at delivery and at the 4- to 12-week postpartum visit was collected from the billing records of the enrolling clinic. Information on insurance during the remainder of the first year postpartum was collected through the outcomes survey at 12 months' postpartum. Anxiety assessment was based on the 3-question subscale of the EPDS, with a score of 6 or greater suggesting anxiety. $22-24$

This report is focused on the women whose baseline PHQ-9 score was not elevated (less than 10). 4,10,25-27 We considered these women to be appropriate for rescreening. The women with elevated initial scores were also sent packets at 6 and 12 months, but their PHQ-9 scores are considered reassessment, ${ }^{26}$ and those results were included in the TRIPPD main outcomes report. ${ }^{4}$

\section{Data Analysis}

Simple percentages were calculated to determine rates of women newly identified to be at high risk of PPD at 6 and 12 months' postpartum. Generalized linear mixedeffects models were used to identify any factors that might predict a high PHQ-9 score at 6 or 12 months' postpartum after a baseline PHQ-9 score of less than 10 . Other choices of cutoff for baseline PHQ-9 had smaller odds ratios. Factors we included in the models were based on previously identified predictors from the literature, limited to factors for which we had data, and on bivariate analyses of the patient-reported demographics. Demographic variables were examined sequentially, and only those found to be significantly predictive on bivariate analysis $(P<.05)$ were retained in the final model. Baseline PHQ-9 was examined as a predictor for later elevated score, and we report the choice of cutoff with greatest odds ratio. The goal of these analyses was to identify any factors that might be useful for tailoring rescreening or limiting rescreening to certain subsets of women.

\section{RESULTS}

In the TRIPPD study, of the 1,801 enrolled women who returned the baseline questionnaire $(78.8 \%$ return rate) 1,432 had a PHQ-9 score of less than 10. These women were defined as having screened PPD negative for depression risk and were therefore eligible for PPD rescreening (Figure 1). Table 1 displays overall demographic information for the 1,432 women, comparing the demographics stratified for women who had an elevated PHQ-9 at either 6 or 12 months' postpartum, those whose PHQ-9 remained at less than 10 on 
both occasions, and the women whose outcome was unknown because they returned the baseline survey packet but neither the 6 - nor 12 -month survey packets.

At 6 months' postpartum, 1,235 women whose screening results for depression were negative (PHQ-9 less than 10) at baseline returned their survey packets ( $86.2 \%$ return rate). Of those, 134 $(10.8 \%)$ reported newly elevated scores of 10 or greater on the PHQ-9. About two-thirds of these women (94) were in the lower elevated score range of 10 to 14 . The remaining women (40) were in the higher PHQ-9 score ranges of 15 or greater and considered to be at very high risk of PPD.

Of the 969 women with prior PHQ-9 scores of less than 10 who returned packets at 12 months (return rate $88.0 \%)$, an additional $6.1 \%$ of the women $(n=59)$ reported newly elevated PHQ-9 scores. Again, most of those scores were in the range of 10 to 14 (48 of the 59,81\%), and 11 (19\%) had PHQ-9 scores of 15 or greater, suggesting very high risk of PPD.

Together the 6- and 12-month repeated screenings yielded 193 women with elevated PHQ-9 scores who had scores of less than 10 on all prior postpartum assessments. In aggregate the 6 - and 12 -month screenings identified $13.5 \%$ of the 1,432 women eligible for rescreening as being at high risk of having postpartum depression (Figure 1).
Factors that were predictive of having a newly elevated PHQ-9 score at 6 or 12 months' postpartum included having a baseline PHQ-9 of anything higher than 1 , a self-reported history of depression, not being married, reporting less than a high school education, and an elevated anxiety screening score at baseline (Table 2). Although the odds ratio for a baseline PHQ-9 score of 2 to 9 to predict future elevated PHQ-9 was 6.7 , this factor has limited value to discriminate between women who will and will not develop an elevated score later, and has a very wide confidence interval. In the models, maternal age, unemployment, the number of other children in the family, living with a partner, household income, and being uninsured at any time during the postpartum period did not predict or protect against increased PHQ-9 scores of or greater than 10, the screening threshold for risk of depression, when the baseline score was less than 10. Suicidal ideation without an elevated PHQ-9 score was also not predictive of future elevated PHQ-9 scores (data not shown).

\section{DISCUSSION}

Repeated screening for PPD using the PHQ-9 at 6 and 12 months' postpartum identified 193 women with newly elevated PPD screening scores after a baseline assessment score of 9 or less. This number represents

Figure 1. Results of repeated administration of PHQ-9 during the first postpartum year.

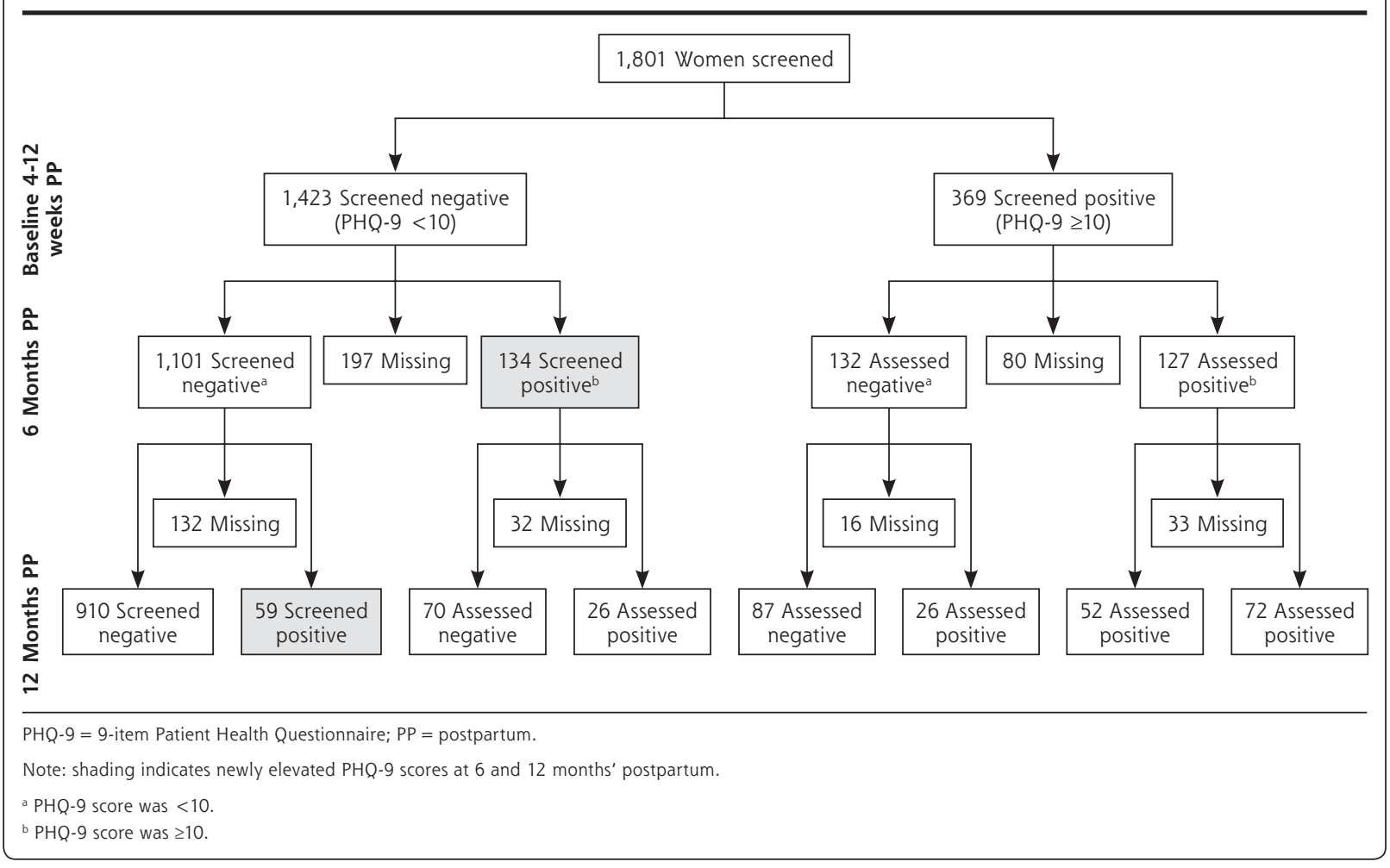


Table 1. Demographic Characteristics of Women Eligible for Repeated Postpartum Depression Screening

\begin{tabular}{|c|c|c|c|c|c|}
\hline Characteristic & $\begin{array}{l}\text { All Women } \\
(n=1,432)\end{array}$ & $\begin{array}{c}\text { Became } \geq 10^{a} \\
(n=193)\end{array}$ & $\begin{array}{l}\text { Remained }<10^{b} \\
(n=910)\end{array}$ & $\begin{array}{l}\text { Unknown } \\
(n=329)\end{array}$ & $P$ Value $^{c}$ \\
\hline Age, mean, y & 26.7 & 25.8 & 27.3 & 25.8 & $<.001$ \\
\hline Married, No. (\%) & $824(57.5)$ & $89(46.1)$ & $594(65.3)$ & $141(42.9)$ & $<.001$ \\
\hline Living with partner, No. (\%) & $1,117(78.0)$ & $141(73.1)$ & $738(81.1)$ & $238(72.3)$ & .02 \\
\hline$\geq 1$ More adult in household, No. (\%) & $1,276(89.1)$ & $164(85.0)$ & $837(92.0)$ & $275(83.6)$ & .004 \\
\hline \multicolumn{6}{|l|}{ Race, No. (\%) } \\
\hline Black & $243(17.0)$ & $33(17.1)$ & $133(14.6)$ & $77(23.4)$ & NS \\
\hline White, not Hispanic & $889(62.1)$ & $124(64.2)$ & $613(67.4)$ & $152(46.2)$ & NS \\
\hline Ethnicity Hispanic & $227(15.9)$ & $25(13.0)$ & $122(13.4)$ & $80(24.3)$ & NS \\
\hline Employed, No. (\%) & $722(50.4)$ & $106(54.9)$ & $457(50.2)$ & $159(48.3)$ & NS \\
\hline > High school, No. (\%) & $781(54.5)$ & $96(49.7)$ & $540(59.3)$ & $145(44.1)$ & .02 \\
\hline \multicolumn{6}{|l|}{ Income, No. (\%) } \\
\hline$>\$ 50,000$ & $305(21.3)$ & $33(17.1)$ & $230(25.3)$ & $42(12.8)$ & .02 \\
\hline$<\$ 20,000$ & $540(37.7)$ & $88(45.6)$ & $285(31.3)$ & $167(50.8)$ & $<.001$ \\
\hline Primiparous, No. (\% & $484(33.8)$ & $58(30.1)$ & $322(35.4)$ & $104(31.6)$ & NS \\
\hline History of depression, No. (\%) & $373(26.0)$ & $87(45.1)$ & $202(22.2)$ & $84(25.5)$ & $<.001$ \\
\hline Uninsured anytime in $1^{\text {st }}$ postpartum year, No. (\%) & $404(28.2)$ & $55(28.5)$ & $269(29.6)$ & $80(24.3)$ & NS \\
\hline Anxiety by EPDS questions 3,4,5, No. (\%) & $125(8.7)$ & $36(18.7)$ & $56(6.2)$ & $33(10.0)$ & $<.001$ \\
\hline \multicolumn{6}{|c|}{ EPDS = Edinburgh Postnatal Depression Scale; NS = nonsignificant at P $\geq .05 ;$ PHQ-9 $=9$-item Patient Health Questionnaire. } \\
\hline \multicolumn{6}{|c|}{ Note: Eligible women were those enrolled in the study who returned baseline PHQ-9 scores of less than 10.} \\
\hline \multicolumn{6}{|c|}{ a Woman whose PHQ-9 scores became elevated at 6 or 12 months. } \\
\hline \multicolumn{6}{|c|}{ b Women whose PHQ-9 scores remained below 10 at 6 and 12 months. } \\
\hline
\end{tabular}

Table 2. Predictors of Later Onset of Elevated Postpartum Depression Screening Scores

\begin{tabular}{lcl}
\hline Predictor & $\boldsymbol{P}$ Value & OR $(95 \% \mathbf{C l})$ \\
\hline PHQ-9 >1 at baseline & $<.001$ & $6.7(3.7,12.2)$ \\
History of depression & $<.001$ & $2.2(1.6,3.0)$ \\
Not married & .04 & $1.4(1.0,2.0)$ \\
$<$ High school graduate & .007 & $1.8(1.2,2.7)$ \\
EPDS anxiety score $\geq 6$ & .002 & $2.0(1.3,3.0)$ \\
\hline
\end{tabular}

EPDS $=$ Edinburgh Postnatal Depression Scale; PHQ-9 = 9-item Patient Health Questionnaire

Note: Generalized linear mixed-effects model predicting high PHQ-9 scores at either 6 months or 12 months' postpartum, after a baseline PHQ-9 score $<10$.

$13.5 \%$ of women whose scores were less than 10 at baseline (4 to 12 weeks' postpartum). New cases were identified at both the 6 -month and the 12 -month rescreening. Although regression analysis did identify some characteristics associated with the likelihood of converting to an elevated screening score (Table 2 ), only the elevated anxiety screening score can be accessible through health care. One might expect women with higher baseline PHQ-9 scores to be at higher risk of later development of PPD. We found, however, that the optimal cutoff score for predicting later elevated screening scores was 2, suggesting that baseline level of depressive symptoms is not useful for predicting later elevated levels.
Several studies reported on use of PPD screening tools to reassess women at 6 months and later. In some studies, it was difficult to ascertain the rates of new PPD diagnoses or screening scores indicative of depression among those who initially had low scores on screening assessments. In addition, many studies reported results for special groups, such as only adolescent mothers or only urban low-income women, thus limiting generalizability to the US population of pregnant women. For example, Peindl et al enrolled only those women with a history of PPD in a previous pregnancy $(\mathrm{n}=50)$, thus excluding all primiparous women, but identified 7 (14\%) of women with a first elevated EPDS score (10 or greater) at or after 6 months' postpartum. ${ }^{14}$ Banti et al enrolled Italian postpartum women in a study in which $50 \%$ of eligible women were screened at baseline, with a high loss to follow-up by 6 months. ${ }^{15}$ Hanusa et al studied 3 different screening tests in adult women $(\mathrm{N}=123)$, but they reported rates of increased risk of depression at 3 months of $10 \%$ and at 6 months of $7 \%$ as the combined rates for all 3 instruments. Of the 3 included tests, 1 had a low specificity that resulted in a screening score suggesting high risk of depression in more than twice as many women as either the PHQ-9 or EPDS scores, making their results difficult to interpret or compare with our data. ${ }^{16}$

Sheeder et al included only adolescents $(\mathrm{N}=204)$ in a high-risk setting, identifying a total of $2(1 \%)$ 
adolescents with scores at high risk of depression on rescreening at 3 and 6 months' postpartum. ${ }^{17}$ Venkatesh et al also enrolled only adolescent mothers $(\mathrm{N}=106)$ and identified $6(6.2 \%)$ with newly elevated EPDS scores (10 or greater) at 6 months. ${ }^{19}$ Chaudron et al enrolled low-income urban women $(\mathrm{N}=35)$ for rescreening and found that $13(41.9 \%)$ had a newly elevated EPDS scores between 3 and 12 months' postpartum..$^{18}$

The study with a population most comparable to ours is that by Gjerdigen et al, who reported on adult women $(\mathrm{N}=506)$ enrolled from family medicine and pediatric practices. ${ }^{20}$ They found no newly elevated screening scores at the 6 -month rescreening but reported that $4.7 \%$ of women without elevated PPD screening scores at baseline had an elevated EPDS or PHQ-9 score at 9 months' postpartum. The investigators noted the previously unreported lack of any newly elevated screening scores suggesting a high risk of depression at 6 months' postpartum, which they suggested might be a honeymoon period for depressive symptoms.

Similar to some other reported studies and unlike the results from Gjerdigen et al, we found newly elevated PHQ-9 scores at 6 months' postpartum (10.5\% of rescreened women) and cannot support the honeymoon hypothesis. Our combined 6- and 12-month rate of elevated test scores on rescreening was $13.5 \%$, nearly 3 times the $4.7 \%$ reported by Gjerdigen et al in the first 9 months' postpartum. The reasons for this difference are not clear but may include differences in initial screening rates (33\% in Gjerdigen vs $88.2 \%$ in TRIPPD) and longer follow-up, as well as differing demographic characteristics of the women in the 2 studies. Comparing Gjerdigen et al's cohort with ours, we had more multiparous women ( $65 \%$ vs $58 \%$ ), we had fewer women with an annual income of more than $\$ 50,000$ ( $21 \%$ vs more than $37 \%$ ), fewer who were employed (50\% vs $64 \%)$, and fewer who were married (58\% vs $65 \%)$, characteristics that have been reported to be associated with higher rates of PPD. ${ }^{1,3,11,12,20,28}$ In addition, we carried our rescreening over the full year postpartum, whereas the Gjerdigen study's final screening was at 9 months' postpartum. ${ }^{4,20,29}$

This study and the others from the literature affirm that rescreening can identify an additional group of women appropriate for careful clinical evaluation of PPD and who may be candidates for depression therapy. The impact of repeated screening on clinical outcomes has not been studied. For family medicine or collaborative care practices ${ }^{30}$ including mental health that integrates infant and maternal care within a single physician or clinic site, ${ }^{3}$ the opportunity to improve outcomes from repeated screening may be appropriate to extrapolate from successful studies of PPD screening programs in primary care. ${ }^{3}$

Success in improving outcomes may be less able to be extrapolated to practices that do not provide on-site adult mental health care. Olson et al ${ }_{1}^{31}$ as well as other studies completed in pediatric practices, ${ }^{17,18}$ suggest that screening at all child care encounters could lead to discussion with the mother regarding the potential impact of depression on the mother, infant development, and maternal-infant relationships. Although such discussion may be of value, its impact on outcomes is unknown, and to date, studies of referral after an initial or repeated screenings have found limited uptake of mental health services by the women referred from pediatric or obstetrical practices. ${ }^{5,32-35} \mathrm{It}$ is therefore necessary to do studies of screening and repeated screening that actually include outcomes to determine whether assessments of risks or benefits can support evidence-based recommendations for rescreening.

The factors associated with new elevation of PHQ-9 scores after a baseline screening score of less than 10 are similar to those associated with elevated scores in the more immediate postpartum period: history of depression, limited education, not being married, and anxiety. ${ }^{3,4,11,12,20}$ We were unable to assess obesity, history of interpersonal violence, or social support, which have also been associated with PPD in some populations. ${ }^{28}$ Although our reported information about predictors is interesting and consistent with the literature, it is the elevated anxiety screening scores that may represent the most actionable of the items. Whether it is possible that identifying and addressing anxiety at the baseline or during the early postpartum period could provide an opportunity to lower the risk of future elevations of depressive symptoms requires further research.

Reassessment of women with previously elevated PHQ-9 scores was not the purpose of this substudy. Even so, within a practice, implementation of rescreening may be easier if the PPD screening tool is given to all postpartum women at 6 and 12 months regardless of initial screening scores. For those with previously elevated scores, rescreening would be considered an important part of monitoring the previously identified high score and response to therapy had PPD been diagnosed. ${ }^{4,26,36}$ Within our study cohort, reassessment at 6 months found that $42.5 \%$ (157 of 369) of women with baseline-elevated PPD screening scores continued to report substantial levels of depressive symptoms (PHQ-9 of 10 or greater). At 12 months, 19.5\% (72 of the original 369) continued to have elevated scores.

Our study has limitations. Not all women returned their baseline, 6-, or 12-month questionnaires, which 
we used for data in this substudy. As reported in the main TRIPPD report, ${ }^{4}$ the women who did and did not return the survey packets differ in some important ways that may have actually underestimated the rates of depression risk on rescreening. For example, women who did not return the 6 - and 12 -month survey packets were more likely to have lower education levels and lower annual incomes, and they were less likely to be married, all of which have been associated with increased risk of PPD. . $^{3,410,11,20,28}$ Our data are based on PPD screening, which is not the same as a clinically confirmed diagnosis of PPD or a major depressive disorder. We therefore cannot report rates of newly identified depression but only rates of women at increased risk of depression.

Our study was conducted in family medicine clinics and therefore includes the $20 \%$ or so of women ${ }^{37,38}$ who chose to seek perinatal care in those settings. These women have similar rates of elevated PPD screening scores at 4 to 12 weeks' postpartum compared with women choosing obstetrician-gynecologists or pediatricians to provide maternity and well-baby care, minimizing the importance of this limitation. ${ }^{3,21,39}$

The strengths of the study include the large sample size and enrollment across 21 practices and 16 states of a group of women with diverse family annual incomes, educational attainment, and insurance status. Only about $62 \%$ of the women reported themselves to be non-Hispanic white, providing information for a racially and ethnically diverse postpartum population.

With 1 in 3 women having an elevated depression screening score during the first year postpartum, the need for continued outcomes and implementation research in postpartum depression is great. For clinics that have an on-site PPD management program in place, repeated PPD screening during the first postpartum year should be considered based on these results. What these results mean for practices that only screen and then refer women with elevated depression scores to off-site mental health resources is not clear. To date such programs have not shown any impact on outcomes related to the initial screening; therefore, in these settings, it is not possible to extrapolate a benefit of repeated screening for postpartum depression.

To read or post commentaries in response to this article, see it online at http://www.annfammed.org/content/13/3/228.

Key words: postpartum depression; screening; PHQ-9; practice-based research

Submitted August 6, 2014; submitted, revised, January 6, 2015; accepted January 21, 2015.

Funding support: Funded by the Agency for HealthCare Research and Quality: R01-HS40471.

\section{References}

1. Gaynes BN, Gavin N, Meltzer-Brody S, et al. Perinatal depression: prevalence, screening accuracy, and screening outcomes. [Summ]. Evid Rep Technol Assess (Summ). 2005;(119):1-8.

2. National Research Council and Institute of Medicine. Committee on Depression, Parenting Practices, and the Healthy Development of Children. Board on Children, Youth, and Families. Division of Behavioral and Social Sciences and Education. Depression in Parents, Parenting, and Children: Opportunities to Improve Identification, Treatment, and Prevention. Washington, DC: The National Academies Press; 2009. http://books.nap.edu/openbook.php?record_id $=12565$. Accessed Dec 30, 2014.

3. Myers ER, Aubuchon-Endsley N, Bastian LA, et al. Efficacy and Safety of Screening for Postpartum Depression. Comparative Effectiveness Review 106. (Prepared by the Duke Evidence-based Practice Center under Contract No. 290-2007-10066-I.) AHRQ Publication No. 13-EHCO64-EF. Rockville, MD: Agency for Healthcare Research and Quality; April 2013. http://www.effectivehealthcare. ahrq.gov/reports/final.cfm.

4. Yawn BP, Dietrich AJ, Wollan P, et al; TRIPPD practices. TRIPPD: a practice-based network effectiveness study of postpartum depression screening and management. Ann Fam Med. 2012;10(4):320-329.

5. Yawn BP, Olson AL, Bertram S, Pace W, Wollan P, Dietrich AJ. Postpartum Depression: Screening, Diagnosis, and Management Programs 2000 through 2010. Depress Res Treat. 2012;2012:363964.

6. Reay R, Matthey S, Ellwood D, Scott M. Long-term outcomes of participants in a perinatal depression early detection program. J Affect Disord. 2011;129(1-3):94-103.

7. American College of Obstetricians and Gynecologists. Committee on Obstetric Practice. Committee opinion no. 453: Screening for depression during and after pregnancy. Obstet Gynecol. 2010;115 (2 Pt 1):394-395.

8. Recommendations of the U.S. Preventive Services Task Force. The Guide to Clinical Preventive Services 2007. Washington, DC: The Agency for Healthcare Research, and Quality (AHRQ). Pub No. 07-05100. Published Sep 2007. http://www.ahrq.gov/clinic/uspstf/ uspstopics.htm. Accessed Apr 5, 2012.

9. American Academy of Pediatrics. Managing Maternal Depression Before and After Birth. Published Oct 25, 2010. https://www. aap.org/en-us/about-the-aap/aap-press-room/pages/ManagingMaternal-Depression-Before-and-After-Birth.aspx.

10. Mishina $H$, Takayama JI. Screening for maternal depression in primary care pediatrics. Curr Opin Pediatr. 2009;21(6):789-793.

11. Georgiopoulos AM, Bryan TL, Wollan P, Yawn BP. Routine screening for postpartum depression. J Fam Pract. 2001;50(2):117-122.

12. Bryan TL, Georgiopoulos AM, Harms RW, Huxsahl JE, Larson DR, Yawn BP. Incidence of postpartum depression in Olmsted County, Minnesota. A population-based, retrospective study. J Reprod Med. 1999;44(4):351-358.

13. Earls MF; Committee on Psychosocial Aspects of Child and Family Health American Academy of Pediatrics. Incorporating recognition and management of perinatal and postpartum depression into pediatric practice. Pediatrics. 2010;126(5):1032-1039.

14. Peindl KS, Wisner KL, Hanusa BH. Identifying depression in the first postpartum year: guidelines for office-based screening and referral. J Affect Disord. 2004;80(1):37-44.

15. Banti S, Mauri M, Oppo A, et al. From the third month of pregnancy to 1 year postpartum. Prevalence, incidence, recurrence and new onset of depression. Results from the Perinatal DepressionResearch and Screening Unit study. Comp Psych. 2011;2011:343-351.

16. Hanusa BH, Scholle SH, Haskett RF, Spadaro K, Wisner KL. Screening for depression in the postpartum period: a comparison of three instruments. [Larchmt]. J Womens Health (Larchmt). 2008;17(4):585-596.

17. Sheeder J, Kabir K, Stafford B. Screening for postpartum depression at well-child visits: is once enough during the first 6 months of life? Pediatrics. 2009;123(6):e982-e988. 
18. Chaudron LH, Kitzman HJ, Szilagyi PG, Sidora-Arcoleo K, Anson E. Changes in maternal depressive symptoms across the postpartum year at well child care visits. Ambul Pediatr. 2006;6(4):221-224.

19. Venkatesh KK, Zlotnick C, Triche EW, Ware C, Phipps MG. Accuracy of brief screening tools for identifying postpartum depression among adolescent mothers. Pediatrics. 2014;133(1):e45-e53.

20. Gjerdingen D, Crow S, McGovern P, Miner M, Center B. Changes in depressive symptoms over 0-9 months postpartum. [Larchmt]. J Womens Health (Larchmt). 2011;20(3):381-386.

21. Spitzer RL, Kroenke K, Williams JB. Validation and utility of a selfreport version of PRIME-MD: the PHQ primary care study. Primary Care Evaluation of Mental Disorders. Patient Health Questionnaire. JAMA. 1999;282(18):1737-1744.

22. Matthey S. Using the Edinburgh Postnatal Depression Scale to screen for anxiety disorders. Depress Anxiety. 2008;25(11):926-931.

23. Ross LE. Gilbert evans SE, Sellers EM, Romach MK. Measurement issues in postpartum depression part 1: anxiety as a feature of postpartum depression. Arch Womens. Ment Health (Lond). 2003;6(1): 51-57.

24. Kabir K, Sheeder J, Kelly LS. Identifying postpartum depression: are 3 questions as good as 10? Pediatrics. 2008;122(3):e696-e702.

25. Gjerdingen D, Katon W, Rich DE. Stepped care treatment of postpartum depression: a primary care-based management model. Womens Health Issues. 2008;18(1):44-52.

26. Löwe B, Kroenke K, Herzog W, Gräfe K. Measuring depression outcome with a brief self-report instrument: sensitivity to change of the Patient Health Questionnaire (PHQ-9). J Affect Disord. 2004;81(1): 61-66.

27. Wickberg B, Hwang CP. Screening for postnatal depression in a population-based Swedish sample. Acta Psychiatr Scand. 1997;95(1): 62-66.

28. Goyal D, Gay C, Lee KA. How much does low socioeconomic status increase the risk of prenatal and postpartum depressive symptoms in first-time mothers? Womens Health Issues. 2010;20(2):96-104.

29. Gjerdingen D, McGovern P, Center B. Problems with a diagnostic depression interview in a postpartum depression trial. J Am Board Fam Med. 2011;24(2):187-193.
30. Katon WJ, Lin EH, Von Korff M, et al. Collaborative care for patients with depression and chronic illnesses. $N$ Engl J Med. 2010;363(27):2611-2620.

31. Olson AL, Dietrich AJ, Prazar G, Hurley J. Brief maternal depression screening at well-child visits. Pediatrics. 2006;118(1):207-216. Accessed May 13, 2014.

32. Kozhimannil KB, Adams AS, Soumerai SB, Busch AB, Huskamp HA. New Jersey's efforts to improve postpartum depression care did not change treatment patterns for women on medicaid. Health Aff (Millwood). 2011;30(2):293-301.

33. Gjerdingen D, Crow S, McGovern P, Miner M, Center B. Postpartum depression screening at well-child visits: validity of a 2-question screen and the PHQ-9. Ann Fam Med. 2009;7(1):63-70.

34. Smith MV, Shao L, Howell H, Wang H, Poschman K, Yonkers KA. Success of mental health referral among pregnant and postpartum women with psychiatric distress. Gen Hosp Psychiatry. 2009;31(2): 155-162.

35. Flynn HA, Henshaw E, O'Mahen H, Forman J. Patient perspectives on improving the depression referral processes in obstetrics settings: a qualitative study. Gen Hosp Psychiatry. 2010;32(1):9-16.

36. Dietrich AJ, Oxman TE, Williams JW Jr, et al. Re-engineering systems for the treatment of depression in primary care: cluster randomised controlled trial. BMJ. 2004;329(7466):602.

37. Phillips RL, Dodoo MS, McCann JL, et al. Report to the Task Force on the Care of Children by Family Physicians. Washington, DC: The Robert Graham Center for Policy Studies in Family Medicine and Primary Care in collaboration with the American Academy of Pediatrics Center for Child Health Research; 2005.

38. Kozhimannil KB, Fontaine P. Care from family physicians reported by pregnant women in the United States. Ann Fam Med. 2013;11(4): 350-354.

39. Yonkers KA, Smith MV, Lin H, Howell HB, Shao L, Rosenheck RA. Depression screening of perinatal women: an evaluation of the healthy start depression initiative. Psychiatr Serv. 2009;60(3): 322-328. 